\title{
Effects of systemic steroids in patients with severe community-acquired pneumonia
}

\author{
C. Garcia-Vidal*, E. Calbo*, V. Pascual*, C. Ferrer*, S. Quintana* and J. Garau*
}

ABSTRACT: The benefit of systemic steroids as adjunctive treatment in patients with severe community-acquired pneumonia (CAP) remains unclear. The present study aimed to evaluate the impact of corticosteroid treatment on mortality in patients with severe CAP.

A retrospective, observational study of a cohort of patients hospitalised with severe CAP, classes IV and V of the Prognostic Severity Index score, was carried out. Information on epidemiological, clinical and laboratory data, and 30-day mortality was collected from medical charts.

Of the 308 patients evaluated, 238 (77\%) were treated with standard antimicrobial therapy and $70(23 \%)$ received both antibiotics and systemic steroids. Clinical characteristics were similar between steroid and nonsteroid groups, except in the prevalence of male sex and the presence of chronic obstructive pulmonary disease. Systemic steroids were independently associated with a decreased mortality (odds ratio 0.287 ; 95\% confidence interval $0.113-0.732$ ), while severity of CAP (2.923; 1.262-6.770) was the only independent factor associated with increased mortality.

Mortality decreased in the patients with severe CAP who received simultaneous administration of systemic steroids along with antibiotic treatment.

Severity of community-acquired pneumonia remains the most important risk factor associated with increased mortality.

KEYWORDS: Community-acquired pneumonia, immune response, immunomodulation, mortality, systemic steroids

ommunity-acquired pneumonia (CAP) is a frequently occurring severe illness, the first infectious cause of death and the sixth cause of overall mortality in the developed world [1]. In Spain, the incidence of CAP in adults ranges $2-10$ cases 1,000 habitants $\cdot \mathrm{yr}^{-1}$. This incidence increases to up 25-35 cases $\cdot 1,000$ habitants $\cdot \mathrm{yr}^{-1}$ in people aged $>70 \mathrm{yrs}$. At least $20 \%$ of patients with CAP will require hospitalisation and, of these, the mortality is $\sim 10-25 \%$, particularly in patients requiring intensive care unit (ICU) admission [2].

Despite more accurate aetiological diagnosis, effective antibiotic therapy and advances in supportive care, mortality rates of CAP remain similar to those reported $>60$ yrs ago [3] at the dawn of the antibiotic era. The study by AUSTRIAN and GOLD [4] showed that deaths occurring within the first 5 days of treatment were not due to failure to eradicate the microorganism; it suggested that they were due to an inappropriate response of the host.

Previous studies have shown increasing local and systemic inflammatory cytokine levels in patients with CAP [5-7] as a first step of the immune response to this infection. A fine balance between cytokine pro-inflammatory and anti-inflammatory activities has been shown to be critical in the host response. In this context, the modulation of the inflammatory response in severe CAP is an appealing concept and remains a matter of debate. Systemic steroids have an inhibitory effect on cytokine production, and have been proposed as a therapeutic option in pulmonary infection and severe sepsis, mainly in patients with adrenocortical insufficiency. However, information is lacking about the outcome of patients with severe CAP treated with both systemic steroids and antibiotics.

The aims of the present study were: 1) to determine predictive factors for mortality in the cohort of patients with severe CAP; and 2) to evaluate the impact on mortality of systemic steroid administration concomitant with antibiotic therapy at the time of CAP diagnosis.

\section{MATERIALS AND METHODS}

\section{Study subjects}

The study was performed at Hospital Mutua de Terrassa (Barcelona, Spain), an acute-care teaching institution with 25,000 admissions per year,
AFFILIATIONS

Services of ${ }^{*}$ Internal Medicine and

*Intensive Care, Hospital Mutua de Terrassa, University of Barcelona, Barcelona, Spain.

CORRESPONDENCE

C. Garcia-Vidal

Service of Infectious Diseases pl.12

Hospital Universitari de Bellvitge C/Feixa Llarga s/n 08907 L'Hospitalet del Llobregat Barcelona

Spain

Fax: 34932607561

E-mail: carolgv75@hotmail.com

Received:

March 082007

Accepted after revision:

July 252007

SUPPORT STATEMENT

The results of the current study were presented, in part, at the 15th European Congress of Clinical Microbiology and Infectious Diseases (Copenhagen, Denmark), April 2005

STATEMENT OF INTEREST

None declared. 
for a population of $\sim 300,000$ inhabitants. The current authors studied all adult patients with CAP who were admitted to hospital between October 1, 2001 and December 31, 2003.

CAP diagnosis was based on the presence of a new infiltrate on chest radiography in a patient with fever and/or leukocytosis when first seen in the Emergency Dept, or in the first $72 \mathrm{~h}$ of admission to hospital. The subset of patients selected were those with severe CAP, as defined by class IV and $\mathrm{V}$ of the prognostic severity index (PSI) reported by FINE et al. [8]. Patients were excluded if: 1) they had been hospitalised within the previous 14 days; 2 ) they were immunosuppressed (chronic use of systemic steroids (methylprednisone $\geqslant 24 \mathrm{mg} \cdot \mathrm{day}^{-1}$ or prednisone $\geqslant 30 \mathrm{mg} \cdot$ day $^{-1} \geqslant 15$ days before the onset of pneumonia), nonsteroid immunosuppressive treatment or HIV); or 3) they had been given a diagnosis of tuberculosis, aspiration or obstructive pneumonia. The study was approved by the Ethical Committee of the current authors' institution.

\section{Study design}

The following variables were retrieved from the patients' medical charts: 1) demographic characteristics (age, sex and residence in a nursing home); 2) comorbidities; 3) clinical data on admission (body temperature, respiratory rate, cardiac frequency and arterial systolic and diastolic blood pressures); 4) laboratory and radiographical findings at admission; 5) PSI scores; 6) aetiology of pneumonia; 7) use of acute systemic corticosteroids; 8) ICU admission; 9) antimicrobial treatment; 10) days from diagnosis to death; and 11) early ( $\leqslant 72 \mathrm{~h}$ ) and 30day mortality. In chronic obstructive pulmonary disease (COPD) patients, spirometry results from the previous $3 \mathrm{yrs}$ were recorded to evaluate severity of disease.

\section{Methods}

Comorbidity was measured using the Charlson Index [9]. This index was used to predict risk of death from comorbid disease. Presence of myocardial infarction, congestive heart failure, peripheral vascular disease, dementia, COPD, autoimmune diseases, peptic ulcer, liver disease, diabetes mellitus, stroke, chronic renal failure, neoplasm, metastatic disease and HIV were included.

Neoplasm, liver disease and diabetes mellitus were specifically evaluated. COPD was defined by a previous clinical diagnosis of COPD, emphysema or chronic bronchitis, and forced spirometry showing forced expiratory volume in one second (FEV1) and FEV1/forced vital capacity $<70 \%$ of their respective reference values. This could represent a possible confounding factor among patients treated with or without systemic steroids and was specifically included in the analysis.

Routine sampling to determine the aetiology of pneumonia included sputum, two sets of blood cultures, and urine for Streptococcus pneumoniae and Legionella pneumophila antigens. Detection of S. pneumoniae antigen in urine was performed by a rapid immunochromatographic assay (Now ${ }^{\mathrm{TM}}$; Binax, Portland, ME, USA). Detection of L. pneumophila serogroup I antigen in urine was performed by an immunoenzymatic commercial method (Legionella Urinary Antigen; Binax). Other additional diagnostic sampling techniques occasionally employed were pleural puncture, tracheobronchial aspirates and bronchoscopy with semiquantitative cultures of bronchoalveolar lavage.
Systemic steroid use was considered when dosages equivalent to methylprednisone $\geqslant 24 \mathrm{mg} \cdot \mathrm{day}^{-1}$ or prednisone $\geqslant 30 \mathrm{mg}$. day $^{-1}$ were given acutely at the time of diagnosis of CAP.

\section{Analysis}

Patients who were placed on systemic steroid therapy concomitant with the diagnosis of severe pneumonia were compared with those not receiving steroids at the time of diagnosis. A second analysis to determine factors related to mortality in the studied population was performed. In the descriptive analysis, percentages were used in categorical variables, mean $\pm \mathrm{SD}$ in continuous variables when normal distribution was assessed by the Kolmogorov-Smirnov test, and median (range) in the others. Univariate analysis was used to detect differences between groups and to identify potential risk factors for mortality. The Chi-squared or Fisher's exact test was used for categorical variables and an unpaired t-test or a nonparametric test (Mann-Whitney U-test) were used for continuous variables. Statistical significance was defined as $p<0.05$. Significant variables in the univariate analysis were further tested by means of logistic regression using the forward conditional method. Presence of COPD was included in the model despite its role as a confounding factor.

\section{RESULTS}

In total, 308 patients were hospitalised with severe CAP during the 26-month period of observation (210 in class IV, 98 in class $\mathrm{V})$. Of these, $68.5 \%$ were male with a mean (range) age of 79.2 (25-100) yrs, and $237(76.9 \%)$ were aged $>70$ yrs. Comorbidities were present in $275(89.2 \%)$ patients, and the Charlson score was 2.49 (0-13). Of the patients, 49 (15.9\%) had malignancy, $26(8.4 \%)$ had liver disease and 61 (19.8\%) were diabetic. There were $97(31.5 \%)$ cases with the diagnosis of COPD who had a mean \pm SD FEV1 of $44.3 \pm 12.96 \%$.

An aetiological diagnosis of CAP was made in 143 (46.4\%) cases. S. pneumoniae was the most common offending microorganism (114 cases, 37\%), followed by L. pneumophila (eight cases, $2.6 \%$ ) and other microorganisms in the remaining 26 cases $(8.4 \%)$.

In total, $18(6 \%)$ patients died. Mortality was eight (3.8\%) out of 210 and $10(10.2 \%)$ out of 98 in class IV and V patients, respectively. Of the patients, 70 received systemic steroids while on antibiotic treatment (47 out of 210 class IV, 23 out of 98 class V). Patients received a median dose of methylprednisolone of $45.7 \mathrm{mg} \cdot \mathrm{day}^{-1}$ or its equivalent, 11.4 (1-34) days from diagnosis. The indications for the administration of systemic steroid therapy are specified in table 1 .

Age, comorbidity, concomitant illness, bacterial aetiology, hypotension and antimicrobial treatment of pneumonia were similar in both groups (table 2).

Male sex $(p<0.001)$ and presence of COPD $(p<0.001)$ were significant variables associated with the systemic steroid treatment group.

It is noteworthy that no differences in mean FEV1 values were found between COPD patients treated with or without corticosteroids. The most frequent reason for acute systemic steroid administration was bronchospasm. Mortality was similar in both groups. 
TABLE 1 Indications for systemic steroid therapy

Indications

Subjects $n$

Broncospasm in chronic obstructive pulmonary disease patients

Broncospasm in patients without previous lung injury

Chronic asthma

Idiopathic pulmonary fibrosis

Unknown

Total

47
14
2
2
5
70

The characteristics of patients with severe CAP who died are shown in table 3 . Only class V of PSI $(p=0.007)$ was a significant risk factor associated with mortality in bivariate analysis (table 3). The current authors' logistic regression model (table 4) showed a protective role of steroid use (odds ratio (OR) 0.287 ; 95\% confidence interval (CI) 0.113-0.732) and confirms severity of pneumonia (OR 2.923, 95\% CI 1.262-6.770) as the only independent factor associated with increased mortality. Specifically, presence of COPD was not associated with a higher mortality rate. There was a trend of higher mortality in patients with the worst FEV1 values; however, differences were not significant.

Early mortality was observed in only two patients; neither had received steroids. Among the patients who died, mean time to death from pneumonia diagnosis was strikingly higher in patients treated with corticosteroids as compared with patients not treated with steroids (13.8 versus 7.1 days, $\mathrm{p}=0.005)$.

\section{DISCUSSION}

In the present study, treatment with systemic steroids reduced mortality in the cohort of patients with severe pneumonia. In fact, the present analysis suggests that patients with severe $\mathrm{CAP}$ receiving corticosteroid therapy and with equal Charlson score, ICU admission rates, monotherapy treatment, aetiology, PSI class and hypotension at admission do better than those not receiving steroid therapy.

The effects of steroids on the immune system are numerous, complex and not well understood [10]; consequently, it is very difficult to evaluate their effects on the immune response against infectious disease. Age, antimicrobial treatment,

\section{TABLE 2 Characteristics of patients treated acutely with systemic steroids and antibiotics}

Acute systemic steroid administration

p-value

No antibiotic treatment Antibiotic treatment

Subjects
Age yrs
Sex male
Charlson index
Comorbidities
COPD
Cancer
Chronic liver disease
Diabetes mellitus
Mean of FEV 1 in COPD patients
ICU admission
CAP treated with monotherapy
CAP aetiology
Streptococcus pneumoniae
Legionella pneumophila
Others
Unknown
Class V of PSI
Hypotension
Mortality

238
$79.3 \pm 10.3$
$151(63)$
$2.32 \pm 1.7$
$50(21)$
$39(16)$
$22(9)$
$45(19)$
$46.4 \pm 13.1$
$9(4)$
$81(34)$
$88(37)$
$7(3)$
$18(8)$
$128(53)$
$74(31)$
$17(7)$
$13(5)$

70

$78.6 \pm 8.84$

$60(85)$

$2.5 \pm 1.6$

$47(67)$

10 (14)

$4(6)$

$16(23)$

$42.8 \pm 12.8$

2 (3)

19 (27)

26 (37)

1 (1)

8 (11)

37 (53)

23 (32)

$3(4)$

$5(7)$
$<0.001$

NS
$<0.001$
NS
$<0.001$
NS
NS
NS
NS
NS
NS
NS

NS
NS
NS

Data are presented as $n$, mean \pm SD or $n(\%)$, unless otherwise stated. COPD: chronic obstructive pulmonary disease; FEV1: forced expiratory volume in one second; ICU: intensive care unit; CAP: community-acquired pneumonia; PSI: prognostic severity index; NS: nonsignificant. 


\begin{tabular}{|c|c|c|c|c|}
\hline \multirow[t]{3}{*}{ TABLE 3} & \multicolumn{4}{|c|}{$\begin{array}{l}\text { Epidemiological characteristics and mortality of } \\
\text { patients with severe community-acquired } \\
\text { pneumonia (CAP) }\end{array}$} \\
\hline & & \multicolumn{2}{|c|}{ Mortality } & \multirow[t]{2}{*}{$p$-value } \\
\hline & & Yes & No & \\
\hline Subjects & & 18 & 290 & \\
\hline Age yrs & & $79.9 \pm 8.5$ & $79 \pm 9.9$ & NS \\
\hline Sex male & & $13(72)$ & 198 (68) & NS \\
\hline Charlson in & & $2.7 \pm 1.9$ & $2.3 \pm 1.7$ & NS \\
\hline \multicolumn{5}{|c|}{ Comorbidities } \\
\hline COPD & & $5(28)$ & 92 (32) & NS \\
\hline Cancer & & $3(17)$ & $46(16)$ & NS \\
\hline Chronic liv & disease & $3(17)$ & $23(8)$ & NS \\
\hline Diabetes $\mathrm{n}$ & |litus & 4 (22) & $57(20)$ & NS \\
\hline Mean of FE & in COPD patients & $37.7 \pm 10.2$ & $44.6 \pm 13.0$ & NS \\
\hline ICU admissi & & $2(11)$ & $9(3)$ & NS \\
\hline CAP treated & vith monotherapy & $7(39)$ & $93(32)$ & \\
\hline CAP aetiolo & & & & NS \\
\hline Streptococ & is pneumoniae & $6(33)$ & $108(35)$ & \\
\hline Legionella & heumophila & $1(6)$ & 7 (3) & \\
\hline Others & & $1(0)$ & $25(9)$ & \\
\hline Unknown & & $10(55)$ & 155 (53) & \\
\hline Class $\mathrm{V}$ of $\mathrm{P}$ & & $10(56)$ & $87(30)$ & 0.007 \\
\hline Hypotensior & & $1(5)$ & $19(7)$ & NS \\
\hline Systemic st & oid treatment & $5(7)$ & 65 (22) & NS \\
\hline
\end{tabular}

Data are presented as $\mathrm{n}$, mean $\pm \mathrm{SD}$ or $\mathrm{n}(\%)$, unless otherwise stated. COPD: chronic obstructive pulmonary disease; FEV1: forced expiratory volume in one second; ICU: intensive care unit; PSI: prognostic severity index; NS: nonsignificant.

steroid dose and its timing, comorbidity and aetiology of pneumonia are important variables to take into account when evaluating the impact of systemic steroids on outcome [11].

In the present cohort, all of these variables were equally represented among the groups. Only prevalence of COPD was significantly higher among patients on systemic steroids. Nevertheless, the mean value of FEV1 was similar between patients with and without steroid treatment. Data on COPD as a risk factor for increased mortality is conflicting [12-15]. It was included in the logistic regression model in order to determine whether COPD could be a confounding factor between mortality and steroid treatment. Even so COPD was not associated with mortality.

Recent studies have demonstrated the efficacy of systemic steroid treatment in patients with septic shock [16] or meningitis [17], but only a few dealing with severe CAP have been reported. Two studies suggest a beneficial effect of systemic steroids in severe CAP, but the characteristics of the population studied are quite different as compared with the present cohort. CONFALONIERI et al. [18] reported that a 7-day course of low-dose hydrocortisone infusion was associated with a significant reduction in duration of mechanical ventilation, length of hospital stay and hospital mortality in patients with CAP admitted to ICU. The inclusion of younger
TABLE 4 Risk factors for mortality in multivariate analysis

OR $(95 \% \mathrm{Cl})$

$\begin{array}{ll}\text { Systemic steroids } & 0.287(0.113-0.732) \\ \text { Severity of CAP } & 2.923(1.262-6.770) \\ \text { COPD } & 3.087(0.906-7.031)\end{array}$

OR: odds ratio; Cl: confidence interval; CAP: community-acquired pneumonia; COPD: chronic obstructive pulmonary disease.

(60.4 versus 77 yrs of age) patients and those with more severe disease (73.9 versus $4 \%$ requiring mechanical ventilation) differed markedly from the current cohort. In this setting, a recent report [19] on cortisol levels in patients with severe CAP admitted to the ICU showed that two-thirds had adrenal insufficiency. Clearly, the presence of underlying adrenal insufficiency could explain the favourable results obtained among some of the patients with severe pneumonia. Likewise, a second study by MONTón et al. [20] assessed the response of modulating therapy in mechanically ventilated patients only. The clinical characteristics of their patients were not reported but, again, severity of disease in these patients was significantly different as compared with the present cohort.

Glucocorticoids inhibit cytokines and other inflammatory molecules stimulated by bacterial infections that could be harmful to the host. However, the use of steroids also exerts a decisive influence in the immune function of macrophages and granulocytes, the main cell host defences against bacteria. It seems clear that precise knowledge of the subset of patients in whom steroids can improve the outcomes is yet to be defined. In this setting, information regarding the effect of corticosteroid use in patients who may have some kind of immunosuppression is lacking. Advances in the knowledge of cytokines release and kinetics, interferon- $\gamma$ and granulocyte colonystimulating factor, the most important cytokines in lung immune response [21-24], will permit a better understanding of the interaction between the endocrine and immune systems in respiratory infection and will make it possible to identify the subset of patients in whom steroid administration would be safe and effective.

The steroid dosage administered to the current patients was not focused on modulating sepsis [25, 26]. Currently, recommended steroid dosages used in sepsis are $50 \mathrm{mg} \cdot 6 \mathrm{~h}^{-1}$ of hydrocortisone [16] (equivalence of one unit of hydrocortisone to five units of methylprednisolone [26]). By chance, the current patients received a median of $45.7 \mathrm{mg} \cdot 24 \mathrm{~h}^{-1}$ of methylprednisolone, which is close to the recommended dose. Nevertheless, hydrocortisone has a more important mineral corticosteroid action. Another difference was the mean number of days on steroid treatment. The present patients were treated for a mean of 11.4 days, while 7 days was the standard duration of steroid treatment in sepsis [18]. This observation might be important because in the present study the patients treated with steroids died significantly later than patients without systemic steroid treatment (13.8 versus 7.1 days, $p=0.005)$. Glucocorticoids exert a decisive influence in macrophage and granulocyte late function and, moreover, steroid 
withdrawal may have a strong "rebound" effect in patients with severe infection [27]. The late mortality in steroid-treated patients suggests that the immune response to resolution of CAP and recovery of lung homeostasis could be seriously compromised by steroid administration at a late stage. A previous study suggests that the inflammatory response is attenuated in patients receiving long-term steroid treatment [28]. Consequently, in future studies to elucidate the role of glucocorticoids in severe $\mathrm{CAP}$, it should be taken into account not only which subset of patients can potentially benefit from its administration, but also the optimal dose and duration of glucocorticoid treatment needed to achieve the proper balance between the beneficial and harmful effects of the inflammatory response.

An important limitation of the current study was that adrenocortical function was not studied in the group of patients treated acutely with systemic steroids. Another limitation was that administration of systemic steroids occurred at different times in the course of the disease. Timing of steroid administration might play a critical role because inflammatory response is a dynamic process and excessive modulation of any pathway could be the cause for an unwanted response. Ascertainment and selection bias, inherent to this design, were not thought to be a significant problem, due to the methodology used to identify patients and the fact that only a small amount of missing data was encountered.

Mortality was lower than expected as predicted by PSI [8]. Better standards of care, including systematic use of the therapeutic guidelines, could at least in partly explain these results. Exclusion of patients with aspiration pneumonia might also have contributed. As reported in a previous study [29], PSI should be considered a severity stratification index but not a good mortality predictor [8].

In conclusion, a better understanding of the interaction between systemic steroids and immune response is necessary before recommending their use in the treatment of severe community-acquired pneumonia. The current results are encouraging and suggest that the effects of systemic steroid administration as immunomodulating agents in an immunocompetent host with severe community-acquired pneumonia can decrease mortality. Further studies are needed to identify the subset of patients with severe pneumonia where systemic steroid administration can be beneficial and to detect the subset of patients in whom the use of steroids could be associated with poor outcomes.

\section{REFERENCES}

1 Statistical Abstract of the United States. 104th Edn. Washington DC, US Government Printing Office, 1984.

2 Almirall J, Bolibar I, Vidal J, et al. Epidemiology of community-acquired pneumonia in adults: a populationbased study. Eur Respir J 2000; 15: 757-763.

3 Evans GM, Gaisford WF. Treatment of pneumonia with 2(p-aminobenzenesulphonamido) pyrine. Lancet 1938; 2: 14-19.

4 Austrian R, Gold J. Pneumococcal bacteremia with special reference to bacteriemic pneumoccoccal pneumonia. Ann Intern Med 1964; 60: 759-770.
5 Puren AJ, Feldman C, Savage N, Becker PJ, Smith C. Patterns of cytokine expression in community-acquired pneumonia. Chest 1995; 107: 1342-1349.

6 Monton C, Torres A, El-Ebiary M, Filella X, Xaubet A, de la Bellacasa JP. Cytokine expression in severe pneumonia: a bronchoalveolar lavage study. Crit Care Med 1999; 27: 1745-1753.

7 Fernandez-Serrano S, Dorca J, Coromines M, Carratala J, Gudiol F, Manresa F. Molecular inflammatory responses measured in blood of patients with severe communityacquired pneumonia. Clin Diagn Lab Immunol 2003; 10: 813-820.

8 Fine MJ, Auble T, Yealy D, et al. A prediction rule to identify low-risk patients with community-acquired pneumonia. N Engl J Med 1997; 336: 243-250.

9 Charlson ME, Pompei P, Ales KL, MacKenzie CR. A new method of classifying prognostic comorbidity in longitudinal studies: development and validation. J Chronic Dis 1987; 40: 373-383.

10 Lionakis M, Kontoyiannis D. Glucocorticoids and invasive fungal infections. Lancet 2003; 362: 1828-1838.

11 Agustí C, Torres A. [Inflammatory response in pneumonia: are glucocorticosteroids useful?] Arch Bronconeumol 2003; 39: 143-145.

12 Fine MJ, Smith MA, Carson CA, et al. Prognosis and outcomes of patients with community-acquired pneumonia. A meta-analysis. JAMA 1996; 275: 134-141.

13 Pallares R, Linares J, Vadillo $\mathrm{M}$, et al. Resistance to penicillin and cephalosporin and mortality from severe pneumococcal pneumonia in Barcelona, Spain. $N$ Engl J Med 1995; 333: 474-480.

14 Ruiz De Ona JM, Gomez Fernandez M, Celdran J, PuenteMaestu L. [Pneumonia in the patient with chronic obstructive pulmonary disease. Levels of severity and risk classification.] Arch Bronconeumol 2003; 39: 101-105.

15 Restrepo MI, Mortensen EM, Pugh JA, Anzueto A. COPD is associated with increased mortality in patients with community-acquired pneumonia. Eur Respir J 2006; 28: 346-351.

16 Keh D, Boehnke T, Weber-Cartens S, et al. Immunologic and hemodynamic effects of "low dose" hydrocortisone in septic shock: a double-blind randomized, placebo controlled crossover study. Am J Respir Crit Care Med 2003; 167: 512-520.

17 De Gans J, van de Beek D, European Dexamethasone in Adulthood Bacterial Meningitis Study Investigators, Dexamethasone in adults with bacterial meningitis. N Engl J Med 2002; 347: 1549-1556.

18 Confalonieri M, Urbino R, Potena A, et al. Hydrocortisone infusion for severe community-acquired pneumonia: a preliminary randomized study. Am J Respir Crit Care Med 2005; 171: 242-248.

19 Salluh J, Verdeal J, Mello G, et al. Cortisol levels in patients with severe community-acquired pneumonia. Intensive Care Med 2006; 32: 595-598.

20 Montón C, Ewing S, Torres A, et al. Role of glucocorticoids on inflammatory response in nonimmunocompromised patients with pneumonia: a pilot study. Eur Respir J 1999; 14: 218-220.

21 Dehoux MS, Boutten A, Ostinelli J, et al. Compartmentalized cytokine production within the human 
lung in unilateral pneumonia. Am J Respir Crit Care Med 1994; 150: 710-716.

22 Boutten A, Dehoux MS, Seta N, et al. Compartmentalized IL-8 and elastase release within the human lung in unilateral pneumonia. Am J Respir Crit Care Med 1996; 153: 336-342.

23 Mullol J, Pujols L, Picado C. [Mechanisms of action of glucocorticoids. Application to the treatment of respiratory inflammation.] Arch Bronconeumol 1996; 32: 527-534.

24 Raño A, Agusti C, Sibila O, Torres A. Associated inflammatory response in pneumonia: role of adjunctive therapy with glucocorticoids. Curr Opin Infect Dis 2006; 19: 179-184.

25 Dellinger RP, Carlet JM, Masur H, et al. Surviving Sepsis Campaign guidelines for management of severe sepsis and septic shock. Crit Care Med 2004; 32: 858-873.

26 Hotchkiss R, Karl I. The pathopysiology and treatment of sepsis. N Engl J Med 2003; 243: 138-150.
27 Schimmer BP, Parker KL. Adrenocorticotropic hormone; adrenocorticol steroids and their synthetic analogs; inhibitors of the synthesis and actions of adrenocorticol hormones. 11th Edn. In: Brunton LL, Lazo JS, Parker KL eds. Goodman and Gilman's: The Pharmacological Basis of Theraspeutics. New York, McGraw Hill: 2006; pp. 1587-1612.

28 Agustí C, Rañó A, Fiella X, et al. Pulmonary infiltrates in patients receiving long-term glucocorticoid treatment. Chest 2003; 123: 488-498.

29 Calbo E, Ochoa de Echagüen A, Rodríguez-Carballeira M, Ferrer C, Garau J. [Hospital admission, duration of stay and mortality in community-acquired pneumonia in an acute care hospital. Correlation between a pneumonia prognosis index and conventional clinical criteria for assessing severity.] Enf Infecc Microbiol Clin 2004; 22: 64-69. 